\title{
Intertechnology Effects in Intelligent Transportation Systems
}

\author{
Seshasai Kanchi, David M. Levinson, and David Gillen
}

The expected benefits of varying combinations of intelligent transportation system applications, such as freeway service patrol, changeable message signs, and ramp metering, were examined. The research analyzed the simulated results of a stylized network in a microscopic traffic simulator. The traffic network included parallel roadways, ramp meters, and changeable message signs. These technologies were tested in various combinations. The effectivenesses were determined, and a measure of intertechnology economies was defined. In brief, additional technologies were found to be subadditive, and more benefits came from each technology in isolation than from the bundling of each with other technologies.

Interest is increasing in understanding the economic viability of intelligent transportation system (ITS) technologies — such as route guidance, freeway service patrols (FSPs), traveler information, and traffic control— to determine their ability to substitute for physical infrastructure investment. Incident management programs are one of the key elements of ITSs. The goal of such programs is to clear incidents and to return traffic flow on the roadway to normal as soon as possible. Incident management programs have been introduced in many places to help reduce the time to detect incidents and their duration. These programs make use of ITS services and coordinate among operating agencies to meet the goals of reduction in the detection and clearance of incidents.

Highway assistance services, also called FSPs, are one of the main approaches used by incident management programs. These services use vehicles to patrol heavily traveled segments and congested sections of freeways that are prone to incidents (1). The main goals of the FSPs are to help identify incident locations, to reduce the duration of incidents, to restore full freeway capacity, and to diminish the risks of secondary accidents to the motorists (2). The role of the patrols is to clear the majority of incidents without any assistance from other agencies. In case of major incidents, the patrols help assess the equipment and resources needed to clear the incidents, coordinate with the other agencies involved, provide the needed traffic control, and act as a buffer between the workers and traffic. They also help detect and verify incidents such as major accidents and pass on the required information to the transportation management centers.

Changeable message signs (CMSs) (also called variable message signs) give information and recommendations to drivers and make them aware of upcoming traffic conditions along their trips. These dynamic messages inform drivers about unexpected inci-

S. Kanchi, ICF Consulting Inc., 9300 Lee Highway, Fairfax, VA 22031. D. M. Levinson, Department of Civil Engineering, University of Minnesota, 500 Pillsbury Drive, SE, Minneapolis, MN 55455. D. Gillen, Institute of Transportation Studies, University of California at Berkeley, 115 McLaughlin Hall, Berkeley, CA 94720. dents and congestion and suggest alternative routes so that delay is minimized.

Ramp-metering programs (RMPs), aimed primarily at recurring congestion, have been applied in numerous cities in the United States as well as throughout the world. Ramp metering aims to limit the number of vehicles entering the freeway from entrance ramps at specific periods of time so that freeway flow can be maintained at a desired service level. Excess demand is forced to wait at the entrance ramp. The intention of ramp metering is, therefore, to maintain uninterrupted, noncongested flow on the freeway as long as possible by transferring delay from the freeway to the entrance ramp. The anticipated consequences of ramp metering include the diversion of traffic to alternative routes in the corridor, less-congested departure times, or even different modes of transportation.

This project aims to evaluate the benefits associated with combinations of these three ITS applications, FSP, CMS, and RMP. The results obtained from each case were analyzed, and the optimal use of ITS technologies was determined. The methodology adopted is described in the form of a flowchart (Figure 1).

\section{MODELING}

This research employs the Advanced Interactive Microscopic Simulator for Urban and Nonurban networks (AIMSUN2) microscopic traffic simulator (3). As other simulators (4), AIMSUN2 continuously models the behavior of each vehicle in the network using carfollowing and lane-changing models. The traffic links or sections in the traffic simulator are represented in the form of channels of pipes for freeways and ramps. The simulator allows users to vary percentages of different vehicles such as cars, trucks, and buses. The input traffic demand is specified by the means of a result container, which encompasses different states and varying traffic flow demands on the basis of time of the day (morning, afternoon, and evening). Rampmetering control is performed by loading a control plan, which contains the details about the traffic flow from the ramps onto the freeway (ramp-metering rate). The modeling system allows adaptive, fixed, and uncontrolled ramp metering with user-defined minimum, initial, and maximum flow metering values. CMSs, which dictate splitting percentages at junctions, can also be modeled directly in AIMSUN2 by inputting the percentage of drivers who will respond to a given message. The optimal CMS splitting percentages for different scenarios is determined by compiling alternative splitting percentages between two paths at the CMS and then choosing the rate that maximizes the overall measure of effectiveness (MOE). FSPs are simulated by generating a traffic incident in the network, which gets cleared after a given time interval determined by the user to reflect the level of service of FSP. The simulator allows the user to create incidents on single or multiple lanes depending on the time when they are activated, the duration (size), and the location in a section of the network. 


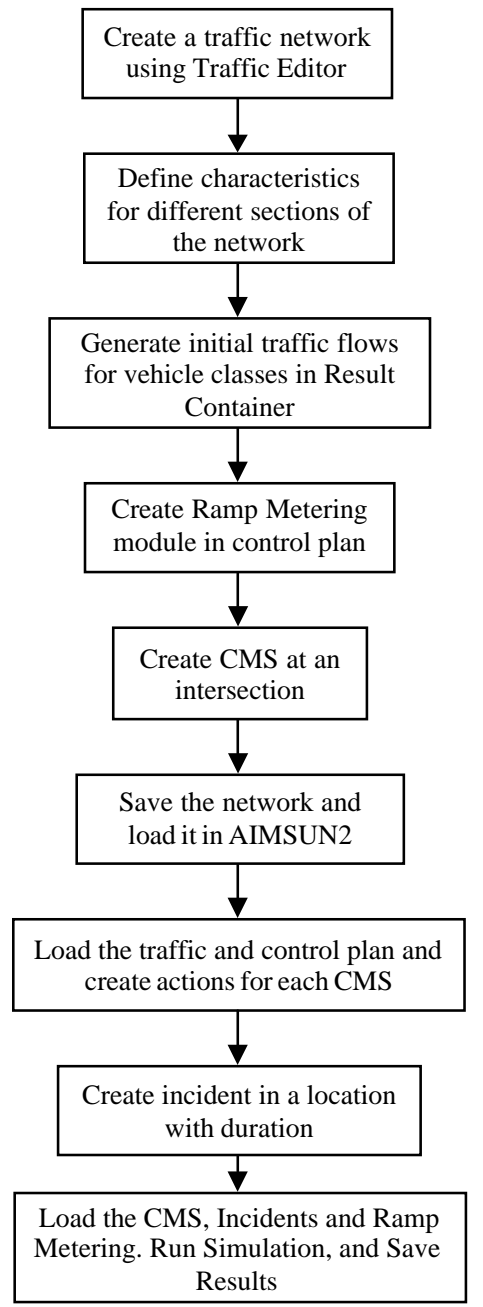

FIGURE 1 Traffic simulation methodology.

The ramp-metering rate optimization process involves the comparison of downstream bottleneck sections for each of two ramps by performing a number of simulations. Each simulation varied the traffic demands and metering rates for the ramps without any incident and thus no need for CMS or FSP. The bottleneck sections were compared using basic traffic characteristics of flow, speed, and density for each of the cases and the results of the analysis are shown in Table 1 . The optimal cases that corresponded to maximum flow were selected as the metered flow rates for the ramps. These metered flows were entered as inputs for the next stage of the analysis. We selected Test G (shown in bold in Table 1) because it resulted in more flow than all of its other counterparts, which is consistent with many ramp-metering strategies. In the analysis shown in Table 1, Section 12 denotes the downstream bottleneck section for the first ramp meter, whereas Section 13 denotes the downstream bottleneck for the second ramp meter. Because of the complexity of the traffic simulation modeling and time constraints, we used a constant optimal metering rate of 600 vehicles/h for all scenarios. Such an assumption would lead to some underestimating of positive impacts of using adaptive ramp metering instead of a constant metering rate. Once the optimal traffic demands with metering rates were determined, the final simulation runs were performed with the integration of CMS and FSP with (or without) optimal ramp-metering rates. We designed on-ramps sufficiently long to allow the total inflow of vehicles entering the network during the simulation cycle at all times and thus accounted for different on-ramp delays for different scenarios.

\section{INPUT CHARACTERISTICS AND ASSUMPTIONS}

The input flow characteristics of the system are shown in Figure 2. The free flow speed of vehicles was taken as $88 \mathrm{~km} / \mathrm{h}$ on freeways and $60 \mathrm{~km} / \mathrm{h}$ on entrance ramps. The flow distribution was assumed to be uniform, with traffic comprising all cars. The initial warm-up time for the simulation was set at $15 \mathrm{~min}$ for an overall $120 \mathrm{~min}$ of the simulation run. The input traffic demand consisted of three states: 1,2 , and 3 . States 1 and 3 corresponded to base flow rates of 8,000 vehicles/h, whereas State 2 carried a flow of 10,000 vehicles/h in the entrance section. All the ramps allowed 600 vehicles/h into the freeway for all the three states. State 1 lasted from 0 to $45 \mathrm{~min}$, State 2 lasted from 45 to $75 \mathrm{~min}$, and State 3 lasted from 75 to $120 \mathrm{~min}$, as shown Figure 2. The traffic incidents were activated after $30 \mathrm{~min}$ of simulation run at a specific fixed location with varying rates of clearance. Optimal ramp-metering rates were determined from initial simulations. However, it was assumed that the optimal rampmetering rates remain unaltered even during incidents downstream of the ramp meters.

The network included parallel roadways, ramp meters, and CMSs. We analyzed multiple or group ramp meters. Figure 3 illustrates the test scenario. A downstream bottleneck on a freeway had two upstream ramp meters with a CMS at the diverge point where the

TABLE 1 Sectional Characteristics of Traffic Flow for Sections 12 and 13 by Varying Ramp-Metering Flow

\begin{tabular}{|cc|ccc|ccc|ccc|}
\hline & & \multicolumn{3}{|c|}{} & \multicolumn{3}{c|}{ SECTION } & \multicolumn{3}{c|}{ SECTION } \\
& & \multicolumn{3}{|c|}{ SYSTEM } & & \multicolumn{3}{c|}{12} & & \multicolumn{3}{c|}{13} \\
\hline & veh/h & veh/h & veh/km & & veh/h & veh/km & $\mathrm{km} / \mathrm{h}$ & veh/h & veh/km & $\mathrm{km} / \mathrm{h}$ \\
& Ramp & Total & Average & $\mathrm{km} / \mathrm{h}$ & Total & Average & Speed & Total & Average & Speed \\
Case & Flow & Flow & Density & Speed & Flow & Density & & Flow & Density & \\
\hline A & 0 & 5823 & 21.13 & 59.38 & 2980 & 16.59 & 79.50 & 2976 & 14.35 & 79.98 \\
B & 100 & 6208 & 22.93 & 59.22 & 3075 & 17.83 & 77.73 & 3171 & 16.78 & 77.25 \\
C & 200 & 6613 & 24.92 & 58.90 & 3195 & 19.57 & 75.00 & 3402 & 19.95 & 73.55 \\
D & 300 & 7000 & 26.91 & 58.42 & 3287 & 21.13 & 72.10 & 3576 & 22.18 & 71.13 \\
E & 400 & 7373 & 28.89 & 57.78 & 3342 & 22.99 & 68.56 & 3727 & 24.54 & 68.08 \\
F & 500 & 7725 & 33.43 & 54.07 & 3445 & 36.16 & 52.14 & 3946 & 30.38 & 61.16 \\
G & $\mathbf{6 0 0}$ & $\mathbf{7 9 7 8}$ & $\mathbf{4 4 . 7 4}$ & $\mathbf{4 4 . 5 8}$ & $\mathbf{3 4 2 3}$ & $\mathbf{4 9 . 9 6}$ & $\mathbf{3 9 . 9 1}$ & $\mathbf{4 0 2 1}$ & $\mathbf{3 3 . 7 4}$ & $\mathbf{5 7 . 7 8}$ \\
H & 700 & 7923 & 47.53 & 42.81 & 3356 & 52.38 & 38.30 & 3976 & 35.29 & 56.01 \\
I & 800 & 7911 & 49.83 & 42.00 & 3341 & 53.13 & 38.46 & 3982 & 34.73 & 56.01 \\
\hline
\end{tabular}




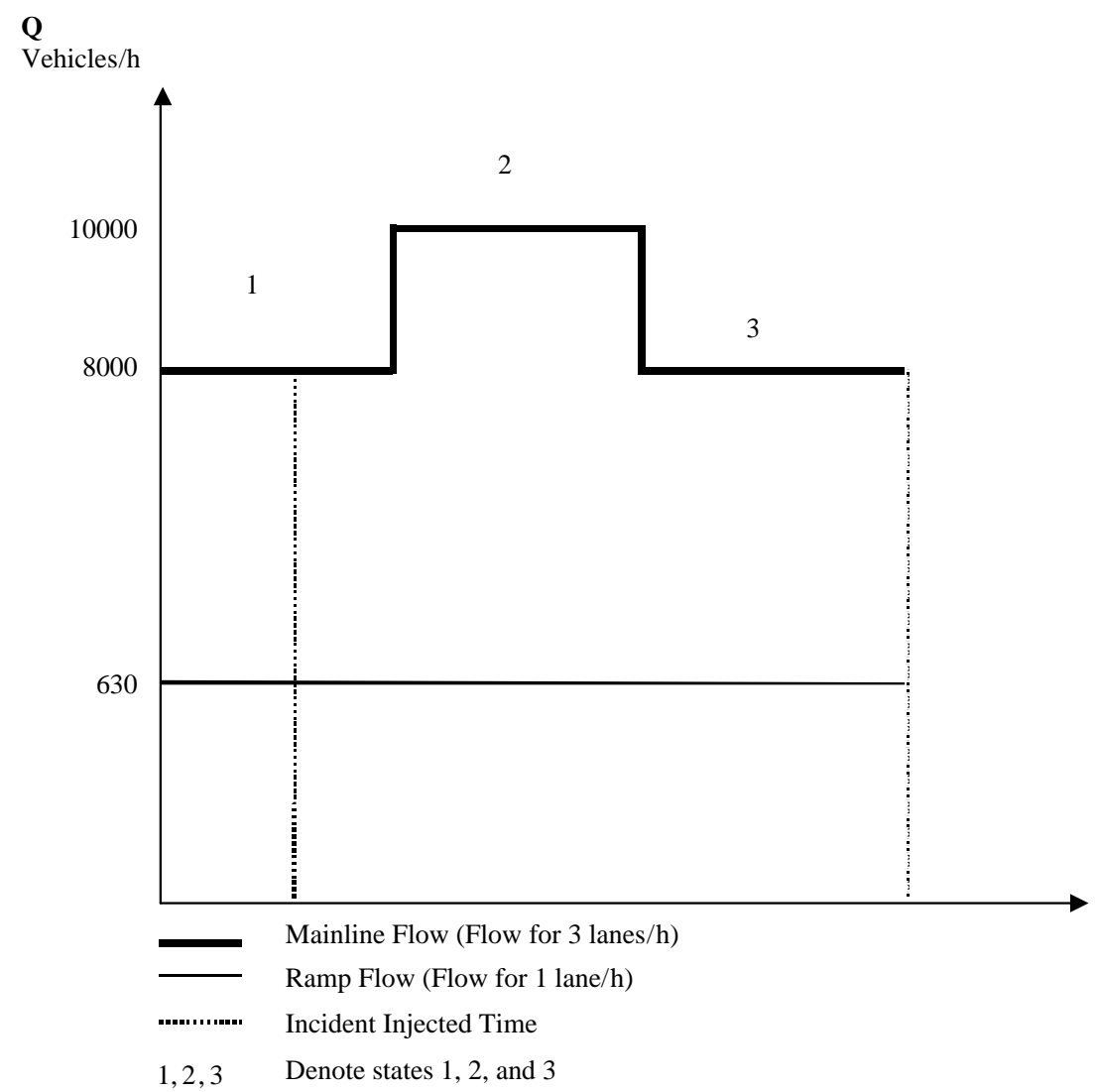

FIGURE 2 Flow characteristic diagram.

freeway with three lanes split into two freeway sections, each of two lanes. The location of the incident was after the second ramp downstream bottleneck, as illustrated in the Figure 3.

The four ramps were tested under both metered and unmetered scenarios. To maintain simplicity for analysis, we restricted ourselves to one type of meter control. We employed CMSs for some scenarios. We specified the proportion of traffic that received the message and the probability of acting on it at the traffic intersection. The evaluation was made for different proportions of people receiving and acting on the message, and we determined how savings change as a result of these different proportions. We assumed that CMSs inform $100 \%$ of drivers, but the percentage that acted based on the message varied from $0 \%$ to $100 \%$. When analyzing CMS, we considered the optimal switching rate for that case. Using different actions, each corresponding to a specific CMS, the turning probability was altered. We believe that with an appropriate severity of message and some experience, turning probabilities can be fine-tuned. FSP is not directly included in the simulator but can be modeled by injecting an incident and accelerating the clearance rate. The blockage can affect one or two lanes, and the clearance rate of the incident can be changed.

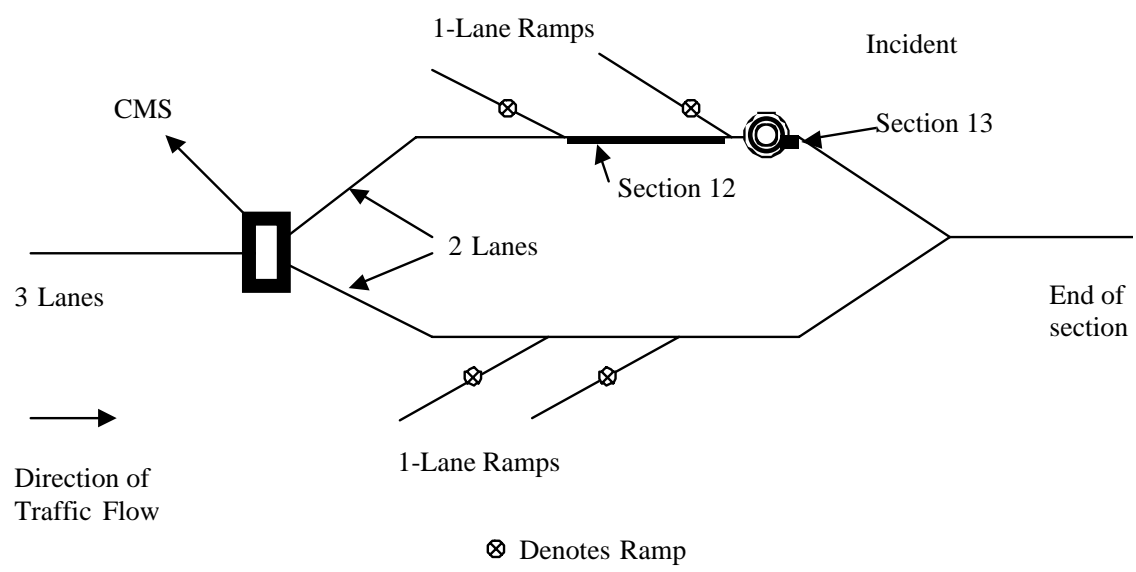

FIGURE 3 Test network. 


\section{MEASURE OF EFFECTIVENESS}

Once the fixed ramp-metering rates were obtained, these values were fed into the microscopic traffic simulator and the analysis was performed with different combinations of CMS turning flow percentages at the diverge point and with different incident durations. To determine the efficiency of our test traffic network, we measured the expected net benefits of some combination of ITS applications.

Inspired by the notion of consumer's surplus, our approach defined an MOE as in Equation 1, illustrated in Figure 4. Figure 4 shows that with the increase in output flow $Q_{s}$ to $Q_{i}$, the travel time decreases from $T_{s}$ to $T_{i}$. The figure represents two sets of conditions, both with fixed demand $D$, one with $S_{s}$ and the other with supply $S_{i}$. The shaded block in the figure represents the change in benefit $(\Delta \beta)$ in vehicle hour units, which is approximated by Equation 1 and which sums up benefit on all links (including ramps) on the network:

$\Delta \beta_{i s}=\sum_{j=1}^{J} \frac{1}{2}\left(Q_{s j}+Q_{i j}\right)\left(T_{s j}-T_{i j}\right)$

where

$T_{s j}$ and $Q_{s j}=$ the fixed section travel time and outflow characteristics that correspond to a standard base case (one- or two-lane incident blockage and an equal split of main-line traffic between the top and bottom roads) for both metering and nonmetering tests on Section $j$,

$T_{i j}$ and $Q_{i j}=$ the sectional travel and flow characteristics for a varying incident duration and turning flow percentages for each Section $j$,

$J=$ the number of sections in the system, and

$\Delta \beta_{i s}=$ how much the system $i$ is better or worse off than system $s$ with regard to vehicle hours for all sections.

Both freeway and ramp sections are included in this analysis.

Although the loaded traffic onto each ramp is fixed, the traffic on each section is not fixed because of queuing and capacity restrictions associated with incidents.

\section{INTERTECHNOLOGY ECONOMIES}

The objective of the paper was to test whether intertechnology economies are present between FSP, CMS, and RMP. Intertechnology economies exist whenever the benefits of all technologies together exceed the sum of benefits of each technology individually. In other words, intertechnology economies are present if the individual benefit of consuming the outputs jointly is greater than the sum of the benefits of consuming the products separately. Thus, if $a, b$, and $c$ are benefits in units of vehicle hours corre-

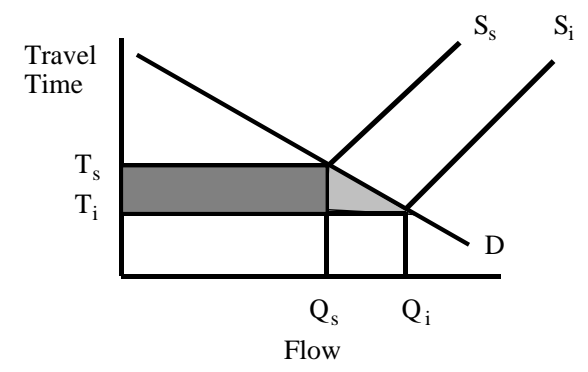

FIGURE 4 Benefit measurement. sponding to FSP, CMS, and RMP in isolation, and $d$ represents overall benefits for consuming all the technologies simultaneously, then an intertechnology economy implies $a+b+c<d$, a case we call superadditive. Otherwise, if $a+b+c>d$, an intertechnology diseconomy is indicated, a case we call subadditive. Using this definition, we examined how the MOE changes when we add ITS technologies for different incident clearance intervals with one- and two-lane blockages.

\section{RESULTS}

First we tested the change in benefit in the presence of ramp metering. As noted earlier, the actual demand on-ramps was set as 630 vehicles/h, whereas the optimal metering flow rate was 600 vehicles/h. Table 2 shows how the benefit changed with additional ITS technologies, which indicates whether the system has a particular ITS technology for one-lane and two-lane blockages. In the case with no CMS, a standard 50\% split between the two routes was assumed; for the cases with no FSP, a standard incident clearance rate of 20 min was assumed. Similarly, in the cases with FSP and CMS, the MOE corresponding to $10 \mathrm{~min}$ incident clearance rate and optimal splitting rates was considered.

In this first case, the change in benefit (in vehicle hours) for each case in one- and two-lane blockages was measured with respect to a standard base case with an incident but no early clearance (i.e., no FSP), no diversion (50\% to 50\%), and standard metering rate of 600 vehicles/h. Table 2 shows the change in benefit for a system with various combinations of FSP, CMS, and RMP for one- and two-lane incident blockages. Table 2 also shows the change in benefit for one-lane blockages is lower than for two-lane blockages, showing that the traffic conditions are more severe and worse in the latter case.

To determine whether FSPs and CMSs are superadditive or subadditive, we must compare the savings attained from each of them separately and together. In other words, we need to find whether

Benefits(FSP, 0) + Benefits $(0$, CMS $) \lessgtr$ Benefits(FSP, CMS $)$

where

$$
\begin{aligned}
\text { Benefits }(\mathrm{FSP}, 0)= & \text { benefits attained only because of FSP, which } \\
& \text { is the difference between Benefits(with FSP, } \\
& \text { without CMS) and Benefits(without FSP, } \\
& \text { without CMS); } \\
\text { Benefits }(0, \mathrm{CMS})= & \text { benefits attained only because of CMS, which } \\
& \text { is the difference between Benefits(with CMS, } \\
& \text { without FSP) and Benefits(without CMS, } \\
& \text { without FSP); and }
\end{aligned}
$$

\begin{tabular}{|c|c|c|c|c|c|}
\hline \multirow[b]{2}{*}{ Cases } & & \multicolumn{2}{|c|}{$\begin{array}{l}1 \text { Lane } \\
\text { Blockage }\end{array}$} & \multicolumn{2}{|c|}{$\begin{array}{l}2 \text { Lane } \\
\text { Blockage }\end{array}$} \\
\hline & & $\begin{array}{l}\text { With } \\
\text { Metering }\end{array}$ & $\begin{array}{l}\text { Without } \\
\text { Metering }\end{array}$ & $\begin{array}{l}\text { With } \\
\text { Metering }\end{array}$ & $\begin{array}{l}\text { Without } \\
\text { Metering }\end{array}$ \\
\hline 1 & No FSP, No CMS & 284 & 0 & 523 & 0 \\
\hline 2 & FSP, No CMS & 348 & 140 & 568 & 402 \\
\hline 3 & No FSP, CMS & 496 & 242 & 716 & 129 \\
\hline 4 & FSP, CMS & 531 & 257 & 734 & 485 \\
\hline
\end{tabular}

TABLE 2 Summary Results from Simulation Runs

NotE: Change in benefit in vehicle hours. 


\section{Benefits(FSP, CMS $)=$ benefits attained by both FSP and CMS together, which is the difference between Benefits(with CMS, with FSP) and Ben- efits(without FSP, without CMS).}

Putting the values for savings in Equation 2, we see from Table 3 that we get [Benefits (FSP , 0) + Benefits $(0, \mathrm{CMS})]>$ Benefits (FSP, CMS $)$ or $(65+212=277>247)$, which demonstrates that the two technologies are subadditive. Similarly, we can perform the analysis in the case of two-lane blockages with ramp metering. Table 4 summarizes the analysis of intertechnology economies with and without FSPs, CMSs, and ramp metering for one- and two-lane blockages. The results obtained were for a 20-min incident that was cleared in 10 min with an FSP operating. The change in benefit obtained for cases without each technology were lower than with that technology, which suggests that all of these technologies have positive benefits in congestion-prone networks. Also, the effect of technology was greater for two-lane blockages than for one-lane blockages because the individual benefits from FSP and CMS were higher for the former case. For both one- and two-lane blockages, the ITS technologies were generally subadditive and generated more benefits separately than together. For the one-lane blockage case, CMS and RMP were subadditive, but for two-lane blockages, they were superadditive. This one case of superadditivity may be because there is more main-line congestion during a two-lane blockage.

\section{CONCLUSIONS}

This research identified and developed a method for measuring the benefits associated with bundles of technologies. This novel analysis of what we call intertechnology economies provides insights into how recurring and nonrecurring traffic congestion can be alleviated with ITS technologies. It suggests that most of the gains can be obtained from whatever technology is deployed first, and that successive gains from additional technologies are smaller than from the first application. We observed that all but one of the cases we modeled demonstrated subadditive benefits. This does not mean that multiple ITS technologies should not be pursued but that the benefit claims of ITS when measured separately must not simply be added together. The more severe the incident, the more useful the ITS technology, either separate or combined. For nonrecurring congestion, FSPs were found to generate significantly more gains than either CMSs or ramp-metering applications. Although many of the benefits may be subadditive, cost savings may be associated with deploying multiple technologies that should also be considered in any complete evaluation.

TABLE 3 Illustrative Intertechnology Economy Matrix (FSP, CMS, RMP) for One-Lane Blockages

\begin{tabular}{lccc}
\hline & With CMS & Without CMS & Savings \\
\hline With FSP & 531 & 348 & 182 \\
Without FSP & 496 & 284 & $\mathbf{2 1 2}$ \\
Savings & 35 & $\mathbf{6 4}$ & 247 \\
\hline
\end{tabular}

$\mathbf{2 1 2}+\mathbf{6 4}=276 \quad>\quad 247=531-284$

NoTE: Change in benefit in vehicle hours compared to baseline case of no ramp metering with one lane blockage.
TABLE 4 Intertechnology Economy (FSP, CMS, RMP) for One- and Two-Lane Blockages

\begin{tabular}{|c|c|c|c|c|}
\hline & \multicolumn{2}{|c|}{1 Lane Blockage } & \multicolumn{2}{|c|}{2 Lane Blockage } \\
\hline Technologies & CS & Result & $\mathrm{CS}$ & Result \\
\hline FSP, 0, RMP & & Subadditive & & Subadditive \\
\hline Benefits(FSP,0,0) & 14( & & 402 & \\
\hline Benefits(0,0,RMP) & 28 & & 523 & \\
\hline Benefits(FSP,0,RMP) & 348 & & 568 & \\
\hline 0,CMS,RMP & & Subadditive & & Superadditive \\
\hline Benefits $(0, \mathrm{CMS}, 0)$ & 242 & & 129 & \\
\hline Benefits(0,0,RMP) & 284 & & 523 & \\
\hline Benefits(0,CMS,RMP) & 496 & & 716 & \\
\hline FSP,CMS,0 & & Subadditive & & Subadditive \\
\hline Benefits(FSP , 0, 0) & 141 & & 401 & \\
\hline $\operatorname{Benefits}(0, \mathrm{CMS}, 0)$ & 242 & & 128 & \\
\hline Benefits(FSP, CMS,0) & 257 & & 484 & \\
\hline FSP,CMS,RMP & & Subadditive & & Subadditive \\
\hline Benefits(FSP, CMS ,0) & 257 & & 485 & \\
\hline Benefits(0,0,RMP) & 284 & & 523 & \\
\hline $\begin{array}{l}\text { Benefits(FSP, CMS,RMP) } \\
\quad \text { (baseline: metering off) }\end{array}$ & 531 & & 734 & \\
\hline FSP,CMS,RMP & & Subadditive & & Subadditive \\
\hline Benefits(FSP ,0, RMP) & 64 & & 45 & 5 \\
\hline Benefits(0,CMS, RMP) & 212 & & 148 & \\
\hline $\begin{array}{l}\text { Benefits(FSP, CMS,RMP) } \\
\quad \text { (baseline: metering on) }\end{array}$ & 247 & & 166 & \\
\hline
\end{tabular}

Note: Change in benefit in vehicle hours. All cases except last assumed baseline with all technologies off $(0,0,0)$ with one- or two-lane blockage. Final case assumed baseline with metering on $(0,0,1)$. Benefits were calculated from Table 2. Incidents 20 min without FSP, 10 min with FSP.

$\mathrm{CS}=$ consumers' surplus.

\section{ACKNOWLEDGMENTS}

The authors thank the California Department of Transportation and the California PATH program at the University of California at Berkeley for providing support as a part of the Measuring Network Effects in ITS project and the University of Minnesota. The authors thank Pavithra Kandadai Parthasarathi for help in analyzing freeway service patrols. The authors also thank Panos Michalopoulos, John Hourdakis, and Koka Muralidhar for helping us to perform efficient traffic simulations with AIMSUN2.

\section{REFERENCES}

1. Freeway Operations Section. Highway Helper Summary Report. Minnesota Department of Transportation, St. Paul, 2000.

2. Fenno, D., and M. Odgen. Freeway Service Patrols: A State of the Practice. In Transportation Research Record 1634, TRB, National Research Council, Washington, D.C., 1998, pp. 28-38.

3. Barcel, J., J. L. Ferrer, and R. Grau. Microscopic Traffic Simulation for ATT Systems Analysis. Departament de Estadstica e Investigacion Operativa, Universitat Politecnica de Catalunya, Barcelona, Spain, 1996.

4. Abdulhai, B., J. Sheu, and W. Recker. Simulation of ITS on the Irvine FOT Area Using Paramics 1.5 Scalable Microscopic Traffic Simulator: Phase I: Model Calibration and Validation. Report UCB-ITS-PRR-99-12. California PATH Research, Richmond, 1999.

Publication of this paper sponsored by Committee on Intelligent Transportation Systems. 